\title{
A NOTE ON GENERALIZED SOLUTIONS OF SINGULAR HAMILTONIAN SYSTEMS
}

\author{
KAZUNAGA TANAKA
}

(Communicated by Hal. L. Smith)

\begin{abstract}
We consider $T$-periodic solutions of singular Hamiltonian systems with weak force

$$
\ddot{q}+\nabla V(q, t)=0,
$$

where $V(q, t) \sim-1 /|q|^{\alpha}$ near $q=0$ with $\alpha \in(0,2)$. In particular, we study some properties of generalized $T$-periodic solutions, which were introduced by Bahri and Rabinowitz.
\end{abstract}

\section{INTRODUCTION}

In this paper we study properties of the generalized solutions of singular Hamiltonian systems which were introduced in [BR]. We consider a Hamiltonian system

$$
\begin{gathered}
\ddot{q}+\nabla V(q, t)=0 \quad \text { in } \mathbf{R}, \\
q(t+T)=q(t) \quad \text { in } \mathbf{R},
\end{gathered}
$$

where $q=\left(q_{1}, q_{2}, \ldots, q_{N}\right) \in \mathbf{R}^{N}(N \geq 3), T>0$, and $V(q, t)$ is a function such that

(V1) $V \in C^{2}\left(\left(\mathbf{R}^{N} \backslash\{0\}\right) \times \mathbf{R}, \mathbf{R}\right)$ is $T$-periodic in $t$;

(V2) $V(q, t)<0$ for all $(q, t)$ and $V(q, t), \nabla V(q, t) \rightarrow 0$ as $|q| \rightarrow \infty$ uniformly in $t$;

(V3) $V(q, t)$ is of the form

$$
V(q, t)=-1 /|q|^{\alpha}+U(q, t),
$$

where $\alpha \in(0,2)$ and $U(q, t) \in C^{2}\left(\left(\mathbf{R}^{N} \backslash\{0\}\right) \times \mathbf{R}, \mathbf{R}\right)$ is $T$-periodic in $t$ and satisfies

$$
\begin{gathered}
|q|^{\alpha-\rho} U(q, t),|q|^{\alpha-\rho+1} \nabla U(q, t),|q|^{\alpha-\rho+2} \nabla^{2} U(q, t), \\
|q|^{\alpha-\rho} U_{t}(q, t) \rightarrow 0 \text { as } q \rightarrow 0 \text { uniformly in } t
\end{gathered}
$$

for some $\rho \in(0, \alpha)$.

Received by the editors November 23, 1992.

1991 Mathematics Subject Classification. Primary 58F05; Secondary 58E05.

Key words and phrases. Periodic solutions, singular Hamiltonian systems, minimax methods. 
In this paper we deal with the case $\alpha \in(0,2)$, that is, the so-called weak force case. The difficulty in proving the existence of periodic solutions of (HS.1)(HS.2) comes from the lack of the Palais-Smale compactness condition for the corresponding functional

$$
I(q)=\int_{0}^{T}\left[\frac{1}{2}|\dot{q}|^{2}-V(q, t)\right] d t .
$$

In [BR] Bahri and Rabinowitz proved the existence of generalized $T$-periodic solutions under weaker conditions

$\left(\mathrm{V}^{\prime}\right) \quad V \in C^{1}\left(\left(\mathbf{R}^{N} \backslash\{0\}\right) \times \mathbf{R}, \mathbf{R}\right)$ is $T$-periodic in $t$;

$\left(\mathrm{V}^{\prime}\right) V(q, t) \rightarrow-\infty$ as $q \rightarrow 0$ uniformly in $t$;

and (V2). Here a generalized $T$-periodic solution means a function $q(t)$ such that

$1^{\circ} q(t)$ enters the singularity 0 on a set $D$ of measure 0 ;

$2^{\circ} q(t) \in C^{2}\left(\mathbf{R} \backslash D, \mathbf{R}^{N}\right)$, and $q(t)$ is $T$-periodic;

$3^{\circ} q(t)$ satisfies (HS) on $\mathbf{R} \backslash D$;

$4^{\circ} q(t) \in H^{1}\left([0, T], \mathbf{R}^{N}\right)$ and $I(q)<\infty$;

$5^{\circ}$ if $V(q, t)$ is independent of $t$, then

$$
\frac{1}{2}|\dot{q}(t)|^{2}+V(q(t)) \equiv \text { const } \quad \text { for } t \in \mathbf{R} \backslash D .
$$

They introduced a perturbation of the potential (essentially as in (0.1) below), applied a suitable minimax argument to the perturbed problems, and obtained a generalized $T$-periodic solution as a limit of classical $T$-periodic solutions of perturbed problems.

In [T1] we estimated the number of collisions of a generalized solution

$$
\nu=\#\{t \in(0, T] ; q(t)=0\}
$$

under the conditions (V1)-(V3) (with $\rho=0)$, and we proved

(i) if $\alpha \in(1,2)$, then $\nu=0$; that is, the generalized solution is in fact a classical solution,

(ii) if $\alpha \in(0,1]$, then $\nu \leq 1$; that is, the generalized solution has at most one collision in its period.

To obtain the above result, we introduced a perturbation of the potential

$$
V_{\varepsilon}(q, t)=V(q, t)-\varepsilon /|q|^{2}
$$

for $\varepsilon \in(0,1]$. First, we obtain a classical solution $q_{\varepsilon}(t)$ of

$$
\begin{gathered}
\ddot{q}+\nabla V_{\varepsilon}(q, t)=0 \text { in } \mathbf{R}, \\
q(t+T)=q(t) \quad \text { in } \mathbf{R} .
\end{gathered}
$$

Second, we pass to the limit as $\varepsilon \rightarrow 0$. As to the number of collisions of generalized solutions, we also refer to [B, C, ST, CS1, CS2, T2].

On the other hand, Beaulieu [B] studied the behavior of generalized solutions precisely and proved that if a generalized solution $q(t)$ enters the singularity 0 at time $t=t_{0} \in(0, T]$, then the limit

$$
a_{ \pm}=\lim _{t \rightarrow t_{0} \pm 0} \frac{q(t)}{|q(t)|} \in S^{N-1}
$$

exists under conditions (V1)-(V3). 
The main purpose of this paper is to study the generalized solutions obtained through (HS.1) $)_{\varepsilon}-\left(\right.$ HS.2) more precisely; we study the angle between $a_{+}$and $a_{-}$. Our main result is

Theorem 0.1. Assume (V1)-(V3). Let $q_{\varepsilon}(t)$ be a T-periodic solution of (HS.1) $\varepsilon_{\varepsilon^{-}}$ (HS.2) that is obtained in [BR]. Assume $q_{\varepsilon}(t) \rightarrow q(t)$ in $L^{\infty}$ as $\varepsilon \rightarrow 0$ (if necessary we extract a subsequence) and $q(t)$ enters the singularity 0 at $t=t_{0}$. Set

and

$$
a_{ \pm}=\lim _{t \rightarrow t_{0} \pm 0} \frac{q(t)}{|q(t)|} \in S^{N-1}
$$

$$
\delta_{\varepsilon}=\min _{t \in(0, T]}\left|q_{\varepsilon}(t)\right|>0, \quad d=\lim _{\varepsilon \rightarrow 0} \frac{\varepsilon}{\delta_{\varepsilon}^{2-\alpha}} \in[0, \infty] .
$$

(If necessary, we extract a subsequence again so that the above limit exists.) Then

(i) $d \leq(2-\alpha)^{2}-1$, and

(ii) the angle between $a_{+}$and $a_{-}$equals $2 \pi \sqrt{1+d} /(2-\alpha)$ modulo $\pi$.

We will give a proof in $\S 1$. In $\S 2$ we assume $\alpha=1,(\mathrm{~V} 1)-(\mathrm{V} 3)$, and

(V4) $V(q, t)$ is independent of $t$ and in a neighbourhood of $0 \in \mathbf{R}^{N}$, $V(q, t)$ is a function of $|q|$, i.e.,

$$
V(q, t)=\phi(|q|) \text { for some } \phi(r):(0, \infty) \rightarrow \mathbf{R}
$$

in a neighbourhood of $0 \in \mathbf{R}^{N}$.

We prove that the generalized solution obtained in the above way is a collisionbrake orbit. More precisely, we prove

Theorem 0.2. Assume (V1)-(V4) and $\alpha=1$. Let $q_{\varepsilon}(t)$ be a solution of (HS.1) $)_{\varepsilon}^{-}$ (HS.2) obtained through a minimax method in [BR]. Suppose $q(t)=\lim _{\varepsilon \rightarrow 0} q_{\varepsilon}(t)$ enters the singularity 0 at $t=t_{0}$. Then

$$
d=\lim _{\varepsilon \rightarrow 0} \frac{\varepsilon}{\delta_{\varepsilon}^{2-\alpha}}=0 \text { and } a_{+}=a_{-} .
$$

Moreover, $q(t)$ is a collision-brake orbit; that is,

$$
q\left(t+t_{0}\right)=q\left(-t+t_{0}\right) \text { for all } t .
$$

Remark 0.1 . Theorem 0.2 is also obtained in [CS1] under a slightly different situation.

For the existence of collision-brake orbits, we refer to [CS2, R].

\section{Proof of Theorem 0.1}

Let $E=H_{T}^{1}\left(\mathbf{R}, \mathbf{R}^{N}\right)$ denote the space of $T$-periodic functions on $\mathbf{R}$ with values in $\mathbf{R}^{N}$ under the norm

$$
\|q\|_{E}^{2}=\int_{0}^{T}|\dot{q}|^{2} d t+\int_{0}^{T}|q|^{2} d t
$$

and let

$$
\Lambda=\{q \in E ; q(t) \neq 0 \text { for all } t\} .
$$

We define for $\varepsilon \in(0,1]$

$$
I_{\varepsilon}(q)=\int_{0}^{T}\left[\frac{1}{2}|\dot{q}|^{2}-V(q, t)+\frac{\varepsilon}{|q|^{2}}\right] d t \in C^{2}(\Lambda, \mathbf{R}) .
$$


Remark 1.1. This functional is slightly different from the functional introduced in [T1], but we can treat it in a similar way (cf. [T2]).

We remark that critical points $q \in \Lambda$ of $I_{\varepsilon}(q)$ correspond to classical $T$ periodic solutions of

$$
\ddot{q}+\nabla V(q, t)+2 \varepsilon q /|q|^{4}=0
$$

Let $q_{\varepsilon}(t)$ be the critical point of $I_{\varepsilon}(q)$ obtained in [BR]. In particular, it satisfies

$$
I_{\varepsilon}\left(q_{\varepsilon}\right) \in[m, M], \quad I_{\varepsilon}^{\prime}\left(q_{\varepsilon}\right)=0, \quad \text { index } I_{\varepsilon}^{\prime \prime}\left(q_{\varepsilon}\right) \leq N-2,
$$

where $m, M>0$ are constants independent of $\varepsilon \in(0,1]$ and index $I_{\varepsilon}^{\prime \prime}\left(q_{\varepsilon}\right)$ is the Morse index at $q_{\varepsilon}$.

As in [T1, T2] we define $\delta_{\varepsilon}=\min _{t \in(0, T]}\left|q_{\varepsilon}(t)\right|>0$ and assume $\left|q_{\varepsilon}(t)\right|$ takes its minimum at $t=t_{\varepsilon}$. We also assume $\delta_{\varepsilon} \rightarrow 0$ as $\varepsilon \rightarrow 0$. We introduce a rescaled function

$$
x_{\varepsilon}(s)=\delta_{\varepsilon}^{-1} q_{\varepsilon}\left(\delta_{\varepsilon}^{(\alpha+2) / 2} s+t_{\varepsilon}\right) \quad \text { for } s \in \mathbf{R} .
$$

We extract a subsequence $\varepsilon_{n} \rightarrow 0$-we still denote it by $\varepsilon$-such that

$$
d=\lim _{\varepsilon \rightarrow 0} \frac{\varepsilon}{\delta_{\varepsilon}^{2-\alpha}} \in[0, \infty] \text { exists. }
$$

If $d=\infty$, we repeat the argument of the proof of [T2, Proposition 4.1, Case 2]. We can see

$$
\operatorname{limindex}_{\varepsilon \rightarrow 0} I_{\varepsilon}^{\prime \prime}\left(q_{\varepsilon}\right)=\infty
$$

and it contradicts (1.2). Therefore, we assume $d<\infty$. Repeating the argument in [T1, T2], we can see

$$
x_{\varepsilon}(s) \rightarrow y_{\alpha, d}(s) \text { in } C_{\mathrm{loc}}^{2}\left(\mathbf{R}, \mathbf{R}^{N}\right),
$$

where $y_{\alpha, d}(s)$ is a solution of

$$
\begin{gathered}
\ddot{y}+\alpha y /|y|^{\alpha+2}+2 d y /|y|^{4}=0 \quad \text { in } t \in \mathbf{R}, \\
y(0)=e_{1}, \quad \dot{y}(0)=\sqrt{2(d+1)} e_{2},
\end{gathered}
$$

where $e_{1}, e_{2} \in \mathbf{R}^{N}$ are suitable vectors satisfying $\left(e_{i}, e_{j}\right)=\delta_{i j}$.

Clearly $y_{\alpha, d}(s) \in \operatorname{span}\left\{e_{1}, e_{2}\right\}$ for all $s \in \mathbf{R}$. We use the polar coordinate and write

$$
y_{\alpha, d}(s)=r_{\alpha, d}(s)\left(e_{1} \cos \theta_{\alpha, d}(s)+e_{2} \sin \theta_{\alpha, d}(s)\right),
$$

where $r_{\alpha, d}(s)>0$ and $\theta_{\alpha, d}(s) \in \mathbf{R}$ with $\theta_{\alpha, d}(0)=0$. Modifying the argument in [T1] slightly, we can see

Proposition 1.1. For $\alpha \in(0,2)$ and $d \in[0, \infty)$,

(i) $s \dot{r}_{\alpha, d}(s)>0$ for all $s \neq 0$ and $r_{\alpha, d}(s) \rightarrow \infty$ as $s \rightarrow \pm \infty$;

(ii) $\dot{\theta}_{\alpha, d}(s)>0$ for all $s \in \mathbf{R}$;

(iii) $\theta_{ \pm}(\alpha, d) \equiv \lim _{s \rightarrow \pm \infty} \theta_{\alpha, d}(s)= \pm \pi \sqrt{1+d} /(2-\alpha)$;

(iv) $\liminf _{\varepsilon \rightarrow 0}$ index $I_{\varepsilon}^{\prime \prime}\left(q_{\varepsilon}\right) \geq(N-2) i(\alpha, d)$, where

$$
i(\alpha, d)=\max \{m \in \mathbf{N} ; m<2 \sqrt{1+d} /(2-\alpha)\} .
$$


Proof. The case $d=0$ is proved in [T1]. We can get the case $d>0$ in a similar way.

Now we can prove the first statement of Theorem 0.1 .

Proof of Theorem 0.1(i). Comparing (1.2) and Proposition 1.1(iv), we get $i(\alpha, d)$ $\leq 1$; that is, $2 \sqrt{1+d} /(2-\alpha) \leq 2$. Therefore, we obtain $d \leq(2-\alpha)^{2}-1$.

In what follows, we prove

Proposition 1.2. $a_{ \pm}=e_{1} \cos \theta_{ \pm}(\alpha, d)+e_{2} \sin \theta_{ \pm}(\alpha, d)$.

We can easily get Theorem 0.1 (ii) from the above proposition. In what follows, we assume $t_{\varepsilon} \rightarrow t_{0}$ as $\varepsilon \rightarrow 0$ and choose a constant $h_{0}>0$ such that

$$
q(t) \neq 0 \text { for all } t \in\left[t_{0}-h_{0}, t_{0}+h_{0}\right] \backslash\left\{t_{0}\right\} \text {. }
$$

We study the behavior of $q_{\varepsilon}(t)$ only in the interval $\left(t_{0}-h_{0}, t_{0}+h_{0}\right)$. To prove Proposition 1.2, we first observe

Lemma 1.3. (i) There is a constant $C_{0}>0$ independent of $\varepsilon \in(0,1]$ such that

$$
\left.\left|\frac{1}{2}\right| \dot{q}_{\varepsilon}(t)\right|^{2}-1 /\left|q_{\varepsilon}(t)\right|^{\alpha}+U\left(q_{\varepsilon}(t), t\right)-\varepsilon /\left|q_{\varepsilon}(t)^{2}\right| \mid \leq C_{0}
$$

for all $\varepsilon$ and $t$.

(ii) There is an $l_{0}>0$ such that $\left|q_{\varepsilon}(t)\right| \leq l_{0}$ implies

$$
\frac{d^{2}}{d t^{2}}\left|q_{\varepsilon}(t)\right|^{2}>0 ;
$$

that is, if $\left|q_{\varepsilon}(t)\right| \leq l_{0}$, then $\left|q_{\varepsilon}(t)\right|$ is increasing (resp. decreasing) for $t>t_{\varepsilon}$ (resp. $t<t_{\varepsilon}$ ).

Proof. (i) We can get (i) as in [T1, Proposition 1.7].

(ii) From (1.1) and (i) we have

$$
\begin{aligned}
\frac{1}{2} \frac{d^{2}}{d \dot{t}^{2}}\left|q_{\varepsilon}\right|^{2} & =\left(\ddot{q}_{\varepsilon}, q_{\varepsilon}\right)+\left|\dot{q}_{\varepsilon}\right|^{2} \\
& \geq \frac{2-\alpha}{\left|q_{\varepsilon}\right|^{\alpha}}-\left(\nabla U\left(q_{\varepsilon}, t\right), q_{\varepsilon}\right)-2 U\left(q_{\varepsilon}, t\right)-2 C_{0} .
\end{aligned}
$$

Thus by (V3) we can choose $l_{0}$ such that

$$
(2-\alpha) /|q|^{\alpha}-(\nabla U(q, t), q)-2 U(q, t)-2 C_{0}>0 \text { for }|q| \leq l_{0} .
$$

Thus we get (ii).

We may assume

$$
\left|q_{\varepsilon}(t)\right| \leq l_{0} \quad \text { for all } t \in\left[t_{0}-h_{0}, t_{0}+h_{0}\right]
$$

for sufficiently small $\varepsilon$.

Next we define

$$
e_{\varepsilon}(t)=\left(\left|q_{\varepsilon}\right|^{2}\left|\dot{q}_{\varepsilon}\right|^{2}-\left(q_{\varepsilon}, \dot{q}_{\varepsilon}\right)^{2}\right)^{1 / 2} \text { for } t \text { and } \varepsilon .
$$

We remark that $e_{\varepsilon}(t)=\left|q_{\varepsilon}(t) \times \dot{q}_{\varepsilon}(t)\right|$ when $N=3$. Using (1.1), straightforward computation gives us

$$
\left|d e_{\varepsilon}(t) / d t\right| \leq\left|q_{\varepsilon}\right|\left|\nabla U\left(q_{\varepsilon}, t\right)\right|
$$


We set

$$
\omega_{\varepsilon}(t)=e_{\varepsilon}(t) /\left|q_{\varepsilon}\right|\left|\dot{q}_{\varepsilon}\right|
$$

Then $\omega_{\varepsilon}(t)=\sin \beta$, where $\beta$ is the angle between $q_{\varepsilon}(t)$ and $\dot{q}_{\varepsilon}(t)$. Using (1.1), (1.6), and (1.7), we get

$$
\begin{aligned}
\frac{d \omega_{\varepsilon}}{d t}(t) & =\frac{\frac{d}{d t} e_{\varepsilon}(t)}{\left|q_{\varepsilon}\right|\left|\dot{q}_{\varepsilon}\right|}-\frac{e_{\varepsilon}(t)}{\left|q_{\varepsilon}\right|^{2}\left|\dot{q}_{\varepsilon}\right|^{2}}\left[\left(\frac{q_{\varepsilon}}{\left|q_{\varepsilon}\right|}, \dot{q}_{\varepsilon}\right)\left|\dot{q}_{\varepsilon}\right|+\left|q_{\varepsilon}\right|\left(\frac{\dot{q}_{\varepsilon}}{\left|\dot{q}_{\varepsilon}\right|}, \ddot{q}_{\varepsilon}\right)\right] \\
& \leq \frac{2\left|\nabla U\left(q_{\varepsilon}, t\right)\right|}{\left|\dot{q}_{\varepsilon}\right|}-\omega_{\varepsilon}(t) \frac{\left(q_{\varepsilon}, \dot{q}_{\varepsilon}\right)}{\left|q_{\varepsilon}\right|\left|\dot{q}_{\varepsilon}\right|}\left[\left|\dot{q}_{\varepsilon}\right|^{2}-\frac{\alpha}{\left|q_{\varepsilon}\right|^{\alpha}}-\frac{2 \varepsilon}{\left|q_{\varepsilon}\right|^{2}}\right] \frac{1}{\left|q_{\varepsilon}\right|\left|\dot{q}_{\varepsilon}\right|} \\
& \leq \frac{2\left|\nabla U\left(q_{\varepsilon}, t\right)\right|}{\left|\dot{q}_{\varepsilon}\right|}-\omega_{\varepsilon}(t) \frac{\left(q_{\varepsilon}, \dot{q}_{\varepsilon}\right)}{\left|q_{\varepsilon}\right|\left|\dot{q}_{\varepsilon}\right|}\left[\frac{2-\alpha}{\left|q_{\varepsilon}\right|^{\alpha}}-2 U\left(q_{\varepsilon}, t\right)-2 C_{0}\right] \frac{1}{\left|q_{\varepsilon}\right|\left|\dot{q}_{\varepsilon}\right|}
\end{aligned}
$$

We assume here $\left(q_{\varepsilon}(t), \dot{q}_{\varepsilon}(t)\right)>0$. Then we get

$$
\begin{aligned}
\frac{d \omega_{\varepsilon}}{d t}(t) \leq\left[-\omega_{\varepsilon}(t) \sqrt{1-\omega_{\varepsilon}(t)^{2}}\left(\frac{2-\alpha}{\left|q_{\varepsilon}\right|^{a}}\right.\right. & \left.-2 U\left(q_{\varepsilon}, t\right)-2 C_{0}\right) \\
& \left.+2\left|q_{\varepsilon}\right|\left|\nabla U\left(q_{\varepsilon}, t\right)\right|\right] \frac{1}{\left|q_{\varepsilon}\right|\left|\dot{q}_{\varepsilon}\right|}
\end{aligned}
$$

Now we have

Proposition 1.4. For any $\eta \in(0,1)$ there are constants $L_{1}>1, l_{1} \in\left(0, l_{0}\right)$, and $\varepsilon_{1} \in(0,1)$ such that for $\varepsilon \in\left(0, \varepsilon_{1}\right]$ and $t \in\left(t_{0}-h_{0}, t_{0}+h_{0}\right)$

$$
q_{\varepsilon}(t) \in B_{l_{1}} \backslash B_{L_{1} \delta_{\varepsilon}} \quad \text { implies }\left|\omega_{\varepsilon}(t)\right|<\eta,
$$

where we use the notation $B_{R}=\left\{x \in \mathbf{R}^{N} ;|x| \leq R\right\}$ for $R>0$.

Proof. First, choose $l_{1} \in\left(0, l_{0}\right)$ such that

$$
-\eta \sqrt{1-\eta^{2}}\left((2-\alpha) /|q|^{\alpha}-2 U(q, t)-2 C_{0}\right)+|q||\nabla U(q, t)|<0
$$

for all $|q| \leq l_{1}$ and $t \in(0, T]$.

For the solution $y_{\alpha, d}(s)$ of $(1.5)$, it is clear that

$$
\frac{\left|y_{\alpha, d}(s)\right|^{2}\left|\dot{y}_{\alpha, d}(s)\right|^{2}-\left(y_{\alpha, d}(s), \dot{y}_{\alpha, d}(s)\right)^{2}}{\left|y_{\alpha, d}(s)\right|^{2}\left|\dot{y}_{\alpha, d}(s)\right|^{2}} \rightarrow 0 \quad \text { as } s \rightarrow \pm \infty
$$

and $\left(y_{\alpha, d}(s), \dot{y}_{\alpha, d}(s)\right)>0$ for sufficiently large $s$. Thus we can choose $s_{0}>0$ such that

$$
\left|\frac{\left|y_{\alpha, d}\left(s_{0}\right)\right|^{2}\left|\dot{y}_{\alpha, d}\left(s_{0}\right)\right|^{2}-\left(y_{\alpha, d}\left(s_{0}\right), \dot{y}_{\alpha, d}\left(s_{0}\right)\right)^{2}}{\left|y_{\alpha, d}\left(s_{0}\right)\right|^{2}\left|\dot{y}_{\alpha, d}\left(s_{0}\right)\right|^{2}}\right|<\frac{\eta}{2} .
$$

We set $L_{1}=2\left|y_{\alpha, d}\left(s_{0}\right)\right|>1$ and $\overline{t_{\varepsilon}}=t_{\varepsilon}+\delta_{\varepsilon}^{(\alpha+2) / 2} s_{0}$. Then

$$
\begin{aligned}
\omega_{\varepsilon}\left(\overline{t_{\varepsilon}}\right) & =\frac{\left|q_{\varepsilon}\left(\overline{t_{\varepsilon}}\right)\right|^{2}\left|\dot{q}_{\varepsilon}\left(\overline{t_{\varepsilon}}\right)\right|^{2}-\left(q_{\varepsilon}\left(\overline{t_{\varepsilon}}\right), \dot{q}_{\varepsilon}\left(\overline{t_{\varepsilon}}\right)\right)^{2}}{\left|q_{\varepsilon}\left(\overline{t_{\varepsilon}}\right)\right|^{2}\left|\dot{q}_{\varepsilon}\left(\overline{t_{\varepsilon}}\right)\right|^{2}} \\
& =\frac{\left|x_{\varepsilon}\left(s_{0}\right)\right|^{2}\left|\dot{x}_{\varepsilon}\left(s_{0}\right)\right|^{2}-\left(x_{\varepsilon}\left(s_{0}\right), \dot{x}_{\varepsilon}\left(s_{0}\right)\right)^{2}}{\left|x_{\varepsilon}\left(s_{0}\right)\right|^{2}\left|\dot{x}_{\varepsilon}\left(s_{0}\right)\right|^{2}} \\
& \rightarrow \frac{\left|y_{\alpha, d}\left(s_{0}\right)\right|^{2}\left|\dot{y}_{\alpha, d}\left(s_{0}\right)\right|^{2}-\left(y_{\alpha, d}\left(s_{0}\right), \dot{y}_{\alpha, d}\left(s_{0}\right)\right)^{2}}{\left|y_{\alpha, d}\left(s_{0}\right)\right|^{2}\left|\dot{y}_{\alpha, d}\left(s_{0}\right)\right|^{2}} \in\left(-\frac{\eta}{2}, \frac{\eta}{2}\right)
\end{aligned}
$$

as $\varepsilon \rightarrow 0$. 
So we can assume that $\omega_{\varepsilon}\left(\overline{t_{\varepsilon}}\right) \leq \frac{\eta}{2}$ for $\varepsilon \in\left(0, \varepsilon_{1}\right]$. We can also assume $\left(q_{\varepsilon}\left(\overline{t_{\varepsilon}}\right), \dot{q}_{\varepsilon}\left(\overline{t_{\varepsilon}}\right)\right)>0$. By Lemma 1.3(ii) we remark that $\left|q_{\varepsilon}(t)\right|$ is increasing in $t>t_{\varepsilon}$ as long as $\left|q_{\varepsilon}(t)\right| \leq l_{1}$.

Arguing indirectly, we assume there is a $t>\overline{t_{\varepsilon}}$ such that

$$
q_{\varepsilon}(t) \in B_{l_{1}} \text { and } \omega_{\varepsilon}(t)=\eta \text {. }
$$

Let $\bar{t}>\overline{t_{\varepsilon}}$ be the smallest $t$ that satisfies (1.10). By (1.8) and (1.9) we get $d \omega_{\varepsilon}(\bar{t}) / d t<0$. This contradicts the definition of $\bar{t}$. Therefore, $\omega_{\varepsilon}(t)<\eta$ for $t \in\left[\overline{t_{\varepsilon}}, t_{0}+h_{0}\right]$ as long as $q_{\varepsilon}(t) \in B_{l_{1}}$. Similarly, we can see $\omega_{\varepsilon}(t)<\eta$ for $t \in\left[t_{0}-h_{0}, t_{\varepsilon}-\delta_{\varepsilon}^{(\alpha+2) / 2} s_{0}\right]$ as long as $q_{\varepsilon}(t) \in B_{l_{1}}$. Since $\left|q_{\varepsilon}\left(\overline{t_{\varepsilon}}\right)\right| / \delta_{\varepsilon} \rightarrow L_{1} / 2$ as $\varepsilon \rightarrow 0$, we can obtain the desired result.

We remark here that

$$
d\left|q_{\varepsilon}(t)\right| / d t=\left(q_{\varepsilon} /\left|q_{\varepsilon}\right|, \dot{q}_{\varepsilon}\right) \geq \sqrt{1-\eta^{2}}\left|\dot{q}_{\varepsilon}\right|
$$

provided $\omega_{\varepsilon}(t) \leq \eta$. From (1.6) we can find $C_{1}, C_{1}^{\prime}>0$ such that

$$
1 /\left|q_{\varepsilon}(t)\right|^{\alpha / 2} \leq C_{1}\left|\dot{q}_{\varepsilon}(t)\right| \leq C_{1}^{\prime}|d| q_{\varepsilon}(t)|/ d t| .
$$

Next we show

Proposition 1.5. For any $\bar{\eta}>0$ there are constants $L_{2}>0, l_{2} \in\left(0, l_{1}\right)$, and $\varepsilon_{2} \in\left(0, \varepsilon_{1}\right)$ such that for $\varepsilon \in\left(0, \varepsilon_{2}\right)$

$$
q_{\varepsilon}(s), q_{\varepsilon}(t) \in B_{l_{2}} \backslash B_{L_{2} \delta_{\varepsilon}}
$$

and

implies

$$
t_{\varepsilon}<s<t<t_{0}+h_{0} \quad\left(\text { or } t_{0}-h_{0}<s<t<t_{\varepsilon}\right)
$$

$$
\left|q_{\varepsilon}(s) /\right| q_{\varepsilon}(s)\left|-q_{\varepsilon}(t) /\right| q_{\varepsilon}(t)||<\bar{\eta} .
$$

Proof. First of all, we have $\omega_{\varepsilon}(t)<\eta$ if $q_{\varepsilon}(t) \in B_{l_{1}} \backslash B_{L_{1} \delta_{\varepsilon}}$. We have

$$
\frac{d}{d t} \frac{q_{\varepsilon}(t)}{\left|q_{\varepsilon}(t)\right|}=\frac{\dot{q}_{\varepsilon}\left|q_{\varepsilon}\right|^{2}-q_{\varepsilon}\left(q_{\varepsilon}, \dot{q}_{\varepsilon}\right)}{\left|q_{\varepsilon}\right|^{3}}
$$

i.e.,

Therefore,

$$
\left|\frac{d}{d t} \frac{q_{\varepsilon}(t)}{\left|q_{\varepsilon}(t)\right|}\right|=\frac{e_{\varepsilon}(t)}{\left|q_{\varepsilon}(t)\right|^{2}}
$$

$$
\left|\frac{q_{\varepsilon}(s)}{\left|q_{\varepsilon}(s)\right|}-\frac{q_{\varepsilon}(t)}{\left|q_{\varepsilon}(t)\right|}\right| \leq \int_{s}^{t}\left|\frac{d}{d \tau} \frac{q_{\varepsilon}(\tau)}{\left|q_{\varepsilon}(\tau)\right|}\right| d \tau=\int_{s}^{t} \frac{e_{\varepsilon}(\tau)}{\left|q_{\varepsilon}(\tau)\right|^{2}} d \tau .
$$

Now we estimate $e_{\varepsilon}(\tau)$. We see for $L>L_{1}$

$$
\begin{aligned}
e_{\varepsilon}\left(t_{\varepsilon}+\delta_{\varepsilon}^{(\alpha+2) / 2)} L\right) / \delta_{\varepsilon}^{(2-\alpha) / 2} & \rightarrow\left|\dot{y}_{\alpha, d}(L) \times y_{\alpha, d}(L)\right| \\
& =\left|\dot{y}_{\alpha, d}(0) \times y_{\alpha, d}(0)\right|=\sqrt{2(d+1)}
\end{aligned}
$$

as $\varepsilon \rightarrow 0$.

Since

$$
\left|d e_{\varepsilon}(t) / d t\right| \leq\left|q_{\varepsilon}(t)\right|\left|\nabla U\left(q_{\varepsilon}, t\right)\right| \leq C_{2} /\left|q_{\varepsilon}\right|^{\alpha-\rho} \text { for some constant } C_{2}>0 \text {, }
$$


we have

$$
\begin{aligned}
\left|e_{\varepsilon}(\tau)\right| & \leq\left|e_{\varepsilon}\left(t_{\varepsilon}+\delta_{\varepsilon}^{(\alpha+2) / 2} L\right)\right|+\int_{t_{\varepsilon}+\delta_{\varepsilon}^{(\alpha+2) / 2} L}^{\tau}\left|\frac{d}{d \tau} e_{\varepsilon}(\tau)\right| d \tau \\
& \leq C_{3} \delta_{\varepsilon}^{(2-\alpha) / 2}+C_{4} \int_{t_{\varepsilon}+\delta_{\varepsilon}^{(\alpha+2) / 2} L}^{\tau} \frac{1}{\left|q_{\varepsilon}\right|^{\alpha-\rho}} d \tau
\end{aligned}
$$

We use (1.12), (1.14), and the remark that $\left|q_{\varepsilon}(t)\right|$ is increasing to get for $\tau \in$ $\left[t_{\varepsilon}+\delta_{\varepsilon}^{(\alpha+2) / 2} L, t_{0}+h_{0}\right]$ and $\left|q_{\varepsilon}(\tau)\right| \leq l$

$$
\begin{aligned}
\left|e_{\varepsilon}(\tau)\right| & \leq C_{3} \delta_{\varepsilon}^{(2-\alpha) / 2}+C_{4}^{\prime} \int_{t_{\varepsilon}+\delta_{\varepsilon}^{(\alpha+2) / 2} L}^{\tau} \frac{1}{\left|q_{\varepsilon}\right|^{\alpha / 2-\rho}} \frac{d}{d \tau}\left|q_{\varepsilon}\right| d \tau \\
& \leq C_{3} \delta_{\varepsilon}^{(2-\alpha) / 2}+C_{4}^{\prime \prime}\left|q_{\varepsilon}(\tau)\right|^{(2-\alpha) / 2+\rho} .
\end{aligned}
$$

Therefore, we get from(1.12) and (1.13) that

$$
\begin{aligned}
\left|\frac{q_{\varepsilon}(s)}{q_{\varepsilon}(s) \mid}-\frac{q_{\varepsilon}(t)}{\left|q_{\varepsilon}(t)\right|}\right| & \leq \int_{s}^{t} \frac{C_{3} \delta_{\varepsilon}^{(2-\alpha) / 2}+C_{4}^{\prime \prime}\left|q_{\varepsilon}\right|^{(2-\alpha) / 2+\rho}}{\left|q_{\varepsilon}\right|^{2}} d \tau \\
& \leq C_{1} \int_{s}^{t} \frac{C_{3} \delta_{\varepsilon}^{(2-\alpha) / 2}+C_{4}^{\prime \prime}\left|q_{\varepsilon}\right|^{(2-\alpha) / 2+\rho}}{\left|q_{\varepsilon}\right|^{(4-\alpha) / 2}} \frac{d}{d \tau}\left|q_{\varepsilon}(\tau)\right| d \tau \\
& \leq C_{1} \int_{L \delta_{\varepsilon}}^{l} \frac{C_{3} \delta_{\varepsilon}^{(2-\alpha) / 2}+C_{4}^{\prime \prime} u^{(2-\alpha) / 2+\rho}}{u^{(4-\alpha) / 2}} d u \\
& =C_{1} C_{3} \int_{L}^{l / \delta_{\varepsilon}} \frac{1}{v^{(4-\alpha) / 2}} d v+C_{1} C_{4}^{\prime \prime} \int_{L \delta_{\varepsilon}}^{l} \frac{1}{u^{1-\rho}} d u \\
& \leq C_{1} C_{3} \int_{L}^{\infty} \frac{1}{v^{(4-\alpha) / 2}} d v+C_{1} C_{4}^{\prime \prime} \int_{0}^{l} \frac{1}{u^{1-\rho}} d u .
\end{aligned}
$$

We can easily see for sufficiently large $L>1$ and sufficiently small $l>0$ that

$$
\begin{aligned}
\left|\frac{q_{\varepsilon}(s)}{\left|q_{\varepsilon}(s)\right|}-\frac{q_{\varepsilon}(t)}{\left|q_{\varepsilon}(t)\right|}\right| \leq \bar{\eta} & \text { if } q_{\varepsilon}(s), q_{\varepsilon}(t) \in B_{l} \\
& \text { and } t_{\varepsilon}+L \delta_{\varepsilon}^{(\alpha+2) / 2}<s<t<t_{0}+h_{0} .
\end{aligned}
$$

Similarly we have

$$
\begin{aligned}
\left|\frac{q_{\varepsilon}(s)}{\left|q_{\varepsilon}(s)\right|}-\frac{q_{\varepsilon}(t)}{\left|q_{\varepsilon}(t)\right|}\right| \leq \bar{\eta} & \text { if } q_{\varepsilon}(s), q_{\varepsilon}(t) \in B_{l} \\
& \text { and } t_{0}-h_{0}<s<t<t_{\varepsilon}-L \delta_{\varepsilon}^{(\alpha+2) / 2} .
\end{aligned}
$$

End of the proof of Proposition 1.2. Let $b_{ \pm}=e_{1} \cos \theta_{ \pm}(\alpha, d)+e_{2} \sin \theta_{ \pm}(\alpha, d)$. For any given $\bar{\eta}>0$ we choose $L_{2}>1, l_{2}>0$, and $\varepsilon_{2}>0$ as in Proposition 1.5 .

Since

$$
y_{\alpha, d}(s) /\left|y_{\alpha, d}(s)\right| \rightarrow b_{+} \quad \text { as } s \rightarrow \infty,
$$

we have for sufficiently large $L>L_{2}$ and sufficiently small $\varepsilon \in\left(0, \varepsilon_{2}\right)$

$$
\left|\frac{q_{\varepsilon}\left(t_{\varepsilon}+\delta_{\varepsilon}^{(\alpha+2) / 2} L\right)}{\left|q_{\varepsilon}\left(t_{\varepsilon}+\delta_{\varepsilon}^{(\alpha+2) / 2} L\right)\right|}-b_{+}\right|<\bar{\eta} .
$$


Setting $s=t_{\varepsilon}+\delta_{\varepsilon}^{(\alpha+2) / 2} L$ in Proposition 1.5, we can see if $q_{\varepsilon}(t) \in B_{l_{2}}$ and $t \in\left(t_{\varepsilon}+\delta_{\varepsilon}^{(\alpha+2) / 2} L, t_{0}+h_{0}\right)$, then

$$
\left|q_{\varepsilon}(t) /\right| q_{\varepsilon}(t)\left|-b_{+}\right|<2 \bar{\eta} .
$$

Therefore, we can see

$$
\left\{q_{\varepsilon}(t) ; t \in\left(t_{\varepsilon}, t_{0}+h_{0}\right)\right\} \cap B_{l_{2}} \subset\left\{x \in B_{l_{2}} ;|x /| x\left|-b_{+}\right|<2 \bar{\eta}\right\} \cup B_{L \delta_{\varepsilon}} .
$$

Letting $\varepsilon \rightarrow 0$, we have

$$
\left\{q(t) ; t \in\left(t_{0}, t_{0}+h_{0}\right)\right\} \cap B_{l_{2}} \subset\left\{x \in B_{l_{2}} ;|x /| x\left|-b_{+}\right| \leq 2 \bar{\eta}\right\} .
$$

Since $\bar{\eta}>0$ is arbitrary, we can see

$$
\lim _{t \rightarrow t_{0}+0} \frac{q(t)}{|q(t)|}=b_{+} .
$$

Similarly, we have

$$
\lim _{t \rightarrow t_{0}-0} \frac{q(t)}{|q(t)|}=b_{-} \text {. }
$$

\section{Proof of Theorem 0.2}

Here, we assume (V1)-(V4) and $\alpha=1$.

Proof of Theorem 0.2. Suppose $q(t)$ is a generalized solution of (HS.1)-(HS.2) which enters the singularity 0 at $t=t_{0}$. By (V4), $q(t)$ is a planar motion and $q(t) \times \dot{q}(t)$ is constant near the singularity 0 by the conservation of angular momentum. Since by $(1.6)$

$$
|q(t) \times \dot{q}(t)| \leq|q(t)||\dot{q}(t)| \leq C|q(t)|^{1-\alpha / 2},
$$

we can easily see $q(t) \times \dot{q}(t)=0$ near $t=t_{0}$; that is, $q(t)$ runs on a line through 0 near $t=t_{0}$. Clearly such a solution is uniquely determined by the values

$$
a=\lim _{t \rightarrow t_{0}+0} \frac{q(t)}{|q(t)|}, \quad H=\frac{1}{2}|\dot{q}(t)|^{2}-\frac{1}{|q(t)|^{\alpha}}+U(q(t)) .
$$

Since $\alpha=1$, we get from Theorem $0.1 d=0$ and

$$
\lim _{t \rightarrow t_{0}+0} \frac{q(t)}{|q(t)|}=\lim _{t \rightarrow t_{0}-0} \frac{q(t)}{|q(t)|} .
$$

Therefore, we have

$$
q\left(-t+t_{0}\right)=q\left(t+t_{0}\right) \quad \text { for } t \in \mathbf{R} .
$$

\section{REFERENCES}

[B] A. Beaulieu, Étude de solutions généralisées pour un systeme hamiltonien avec potentiel singulier, Duke Math. J. 67 (1992), 21-37.

[BR] A. Bahri and P. H. Rabinowitz, A minimax method for a class of Hamiltonian systems with singular potentials, J. Funct. Anal. 82 (1989), 412-428.

[C] V.Coti Zelati, Periodic solutions for a class of planar, singular dynamical systems, J. Math. Pures Appl. (9) 68 (1989), 109-119.

[CS1] V. Coti Zelati and E. Serra, Collisions and non-collisions for a class of Keplerian-like dynamical systems, preprint. 
[CS2] _ Multiple brake orbits for some classes of Hamiltonian systems, preprint.

[R] P.H. Rabinowitz, A note on periodic solutions of prescribed energy for singular Hamiltonian systems, preprint.

[ST] E. Serra and S.Terracini, Noncollision solutions to some singular minimization problems with Keplerian-like potentials, preprint.

[T1] K. Tanaka, Non-collision solutions for a second order singular Hamiltonian system with weak force, Ann. Inst. H. Poincaré Anal. Non Linéaire 10 (1993), 215-238.

[T2] _ A prescribed energy problem for a singular Hamiltonian system with a weak force, $\mathrm{J}$. Funct. Anal. 113 (1993), 351-390.

Department of Mathematics, School of Science, Nagoya University, Chikusa-ku, NAGOYA 464, JAPAN

E-mail address: tanaka@math.nagoya-u.ac.jp 